Article

\title{
New System for Measuring Cosmogenic Ne in Terrestrial and Extra-Terrestrial Rocks
}

\author{
Domokos Györe *(D), Luigia Di Nicola, David Currie and Finlay M. Stuart
}

Scottish Universities Environmental Research Centre (SUERC), East Kilbride G75 0QF, UK;

Luigia.DiNicola@glasgow.ac.uk (L.D.N.); d.currie.1@research.gla.ac.uk (D.C.); fin.stuart@glasgow.ac.uk (F.M.S.)

* Correspondence: Domokos.Gyore@glasgow.ac.uk

\begin{abstract}
Cosmogenic Ne isotopes are used for constraining the timing and rate of cosmological and Earth surface processes. We combined an automated gas extraction (laser) and purification system with a Thermo Fisher ARGUS VI mass spectrometer for high through-put, high precision Ne isotope analysis. For extra-terrestrial material with high cosmogenic Ne concentrations, we used multi-collection on Faraday detectors. Multiple measurements $(\mathrm{n}=26)$ of $1.67 \times 10^{-8} \mathrm{~cm}^{3}$ air-derived ${ }^{20} \mathrm{Ne}$ yielded an uncertainty of $0.32 \%$, and ${ }^{21} \mathrm{Ne} /{ }^{20} \mathrm{Ne}=0.17 \%$ and ${ }^{22} \mathrm{Ne} /{ }^{20} \mathrm{Ne}=0.09 \%$. We reproduced the isotope composition of cosmogenic $\mathrm{Ne}$ in the Bruderheim chondrite and Imilac pallasite in a sub-ten mg sample. For lower Ne amounts that are typical of terrestrial samples, an electron multiplier detector was used in peak jumping mode. Repeated analysis of $3.2 \times 10^{-11} \mathrm{~cm}^{3}$ STP ${ }^{20} \mathrm{Ne}$ from air reproduced ${ }^{21} \mathrm{Ne} /{ }^{20} \mathrm{Ne}$ and ${ }^{22} \mathrm{Ne} /{ }^{20} \mathrm{Ne}$ with $1.1 \%$ and $0.58 \%$, respectively, and ${ }^{20} \mathrm{Ne}$ intensity with $1.7 \%(\mathrm{n}=103)$ over a 4-month period. Multiple $(\mathrm{n}=8)$ analysis of cosmogenic Ne in CREU-1 quartz yielded $3.25 \pm 0.24 \times 10^{8}$ atoms $/ g(2 \mathrm{~s})$, which overlaps with the global mean value. The repeatability is comparable to the best data reported in the international experiments performed so far on samples that are $2-5 \times$ smaller. The ability to make precise Ne isotope determinations in terrestrial and extra-terrestrial samples that are significantly smaller than previously analysed suggests that the new system holds great promise for studies with limited material.
\end{abstract}

Keywords: cosmogenic ${ }^{21} \mathrm{Ne}$; noble gas isotope; mass spectrometry; neon isotope; ${ }^{21} \mathrm{Ne}$; $\mathrm{Ne}$ dating; Bruderheim chondrite; Imilac pallasite; CREU quartz; cosmogenic nuclides

\section{Introduction}

Cosmogenic Ne was proven to be an adept recorder of the timing and rates of surface processes on the Earth and Moon [1-3] and the time of meteorite release from parent bodies [4]. Precise determination of neon isotopes in rocks and minerals are conventionally made using static gas magnetic sector mass spectrometers [5-8]. The low production rate at the Earth's surface and the presence of isobaric interferences at all Ne isotopes means that precise determination of terrestrial cosmogenic $\mathrm{Ne}$ is routinely measured in only a handful of laboratories worldwide [5]. Improvements in mass spectrometry in the last ten years, in particular the ability to resolve some of main isobaric interferences [8-10], will lead to better and faster cosmogenic Ne determinations.

Here, we report the use of a Thermo Fisher ARGUS VI mass spectrometer with an automated gas extraction and purification system for the determination of cosmogenic Ne in both extra-terrestrial and terrestrial material. It is a low resolution instrument [11], which requires low background levels and a good understanding of isobaric interferences [6]. Where these can be obtained, the high sensitivity and good instrument stability combine to allow high throughput cosmogenic Ne determinations on samples that are analysed by conventional instruments. Two protocols can be applied depending on Ne concentrations: multi-collection using Faraday detectors and peak-jumping mode using a compact discrete dynode (CDD) detector. We demonstrate the multi-collection Faraday technique with new analysis of cosmogenic Ne in sub-ten mg samples of Bruderheim chondrite and Imilac 
pallasite and the peak-jumping CDD analysis of $19.9 \mathrm{mg}$ aliquots of CREU-1 quartz. We use this study to demonstrate how significant reduction in sample size affects uncertainty and the implication of that in cosmogenic Ne dating.

\section{Analytical System}

The Thermo Fisher ARGUS VI is a low resolution $(\mathrm{R}<200)$, small volume (0.7 litre) 6-detector static vacuum mass spectrometer designed primarily for Ar isotope analysis [12]. It was recently shown to be capable of making high precision determinations of $\mathrm{Ne}, \mathrm{Kr}$ and Xe isotopes $[6,11,12]$. The need to minimise isobaric interferences for Ne isotope determinations demanded several modifications to the mass spectrometer vacuum envelope. Gas equilibrated with the mass spectrometer is forced to enter the ion source via a SAES GP50 ZrAl alloy getter held at room temperature in order to minimise the $\mathrm{H}_{2}{ }^{+}$ level, which controls the rate of formation of $\mathrm{Ar}^{2+}$ and $\mathrm{CO}_{2}{ }^{2+}$ [13], as well as ${ }^{20} \mathrm{NeH}^{+}$[6]. A charcoal-filled finger on the source block is cooled with liquid nitrogen during analysis to reduce the level of residual $\mathrm{Ar}$ and $\mathrm{CO}_{2}$ in the mass spectrometer.

The ion source operates at $4.5 \mathrm{kV}$ acceleration potential, $250 \mu \mathrm{A}$ trap current and $80 \mathrm{eV}$ electron energy to minimise $\mathrm{NeH}^{+}$production in the source [6]. The source parameters (ion repeller voltage, extraction, focus and symmetry) were tuned for maximum sensitivity using ${ }^{20} \mathrm{Ne}$. The magnet position was set to achieve the coincidence of the flat-topped peaks of all Ne isotopes $\left({ }^{20} \mathrm{Ne},{ }^{21} \mathrm{Ne}\right.$ and $\left.{ }^{22} \mathrm{Ne}\right)$ on the Faraday detectors $\left(10^{12} \mathrm{Ohm}\right.$ resistance amplifiers), and of ${ }^{22} \mathrm{Ne}$ and ${ }^{21} \mathrm{Ne}$ on the combination of Faraday and a compact discrete dynode (CDD) detector, respectively (Figure 1). The release of $\mathrm{CO}_{2}$ from the $\mathrm{CDD}$ requires that it is conditioned. In the initial phase of this work, the $\mathrm{CDD}$ was bombarded by a $\mathrm{CO}_{2}$ beam and pumped by the mass spectrometer ion pump for several weeks. This resulted in a reduction in the dynamic $\mathrm{CO}_{2}$ beam intensity from $>100 \mathrm{fA}$ to a normal operating level of $2.5 \mathrm{fA}$.

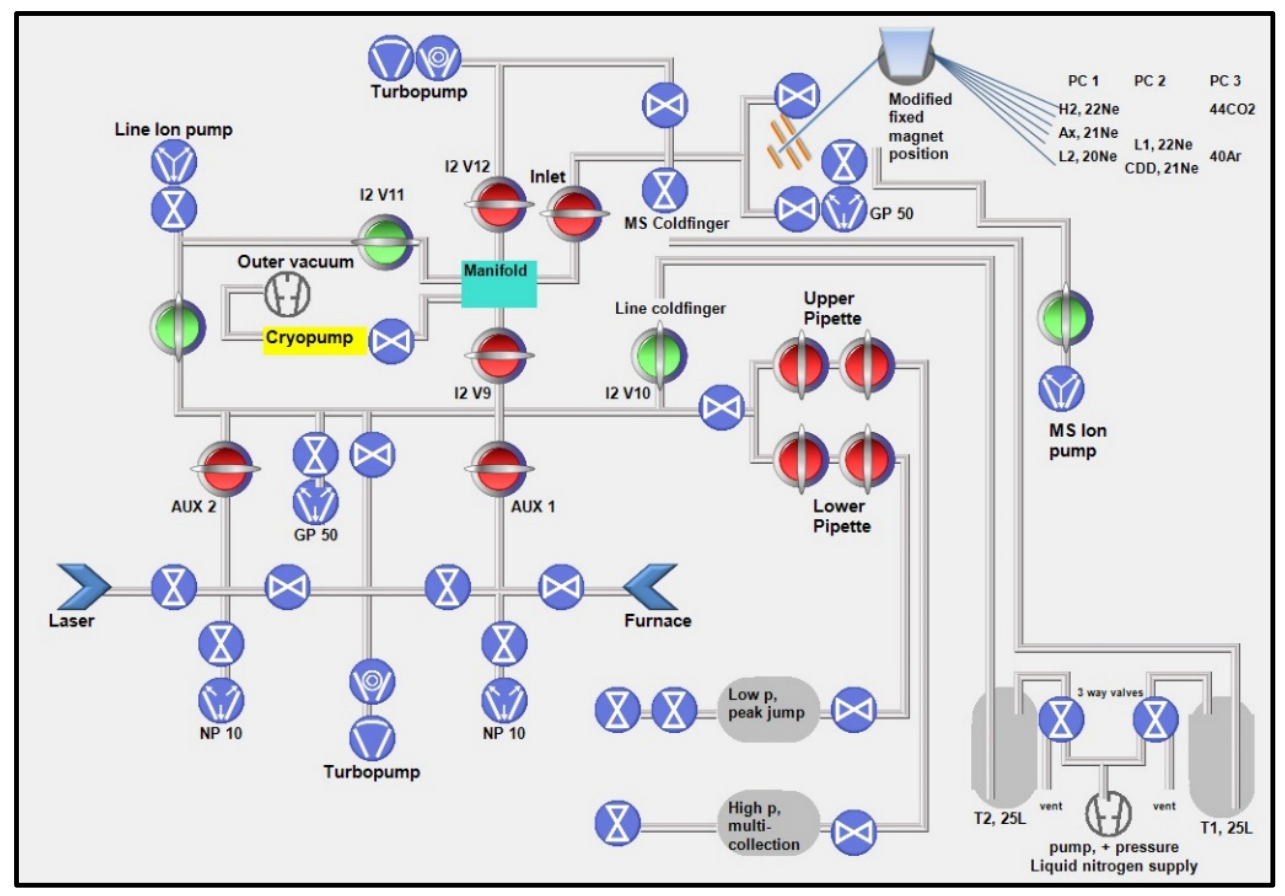

Figure 1. Schematic picture of the fully automatized Thermo Fisher ARGUS VI mass spectrometer and preparation system in SUERC. Two 2L air reservoirs, each equipped with a $0.1 \mathrm{~cm}^{3}$ gas pipette, provided the means for calibration. The magnet position allowed multi collecting of $\mathrm{Ne}$ isotopes. PC1: (H2: $\left.{ }^{22} \mathrm{Ne}-\mathrm{Ax}:{ }^{21} \mathrm{Ne}-\mathrm{L} 2:{ }^{20} \mathrm{Ne}\right)$. PC2: (L1: $\left.{ }^{22} \mathrm{Ne}-\mathrm{CDD}:{ }^{21} \mathrm{Ne}\right)$. PC3: $\left(\mathrm{H} 2:{ }^{44} \mathrm{CO}_{2}-\mathrm{L} 2:{ }^{40} \mathrm{Ar}\right)$. Blue valve: manual. Green: pneumatically actuated, open. Red: pneumatically actuated, closed. PC: peak coincidence. 
High precision Ne isotope analysis is complicated by isobaric interferences at all peaks. Although new instruments can either fully or partially resolve some of the common interferences [8,9], in many cases, a correction is required $[6,7,10,14,15]$. This requires the measurement of several other peaks, which has an implication on measurement time and data precision. The optimisation of analysis procedures in mass spectrometer control software (Qtegra) required the use of four lab books, starting with hydrogen, followed by mass 18, 19, 40 and 44, prior to the analysis of Ne isotopes (Figure 2). The number of cycles required for $\mathrm{Ne}$ isotope analysis was optimised to overcome uncertainty introduced by the time delay. We developed both a multi-collection mode using the array of Faraday detectors where the smallest $\mathrm{Ne}$ beam was greater than $\sim 7 \mathrm{fA}$ and a peak jumping mode that used the CDD for smaller Ne beam intensities.

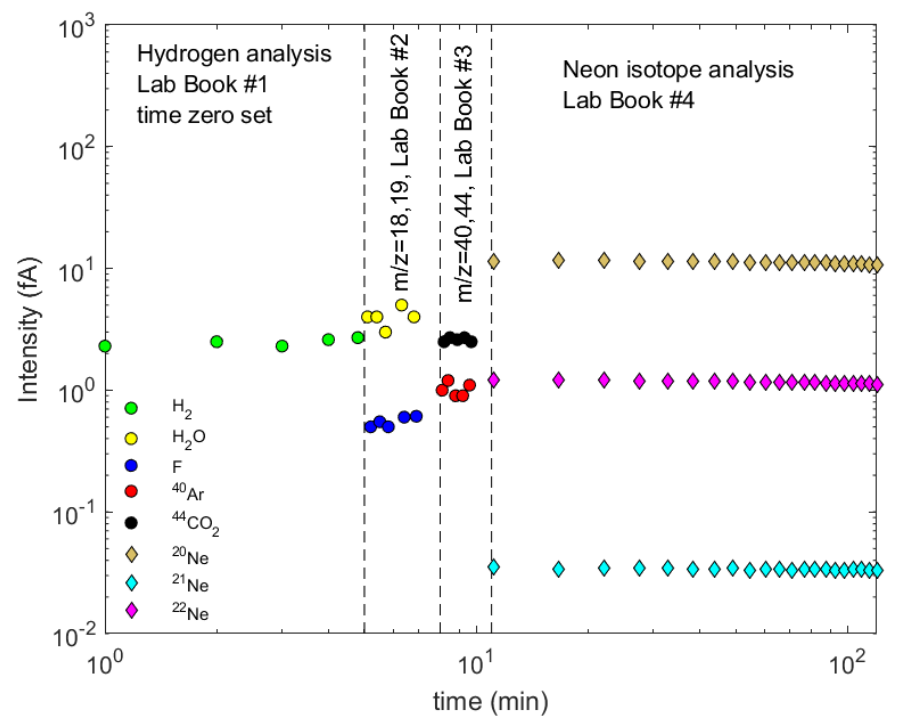

Figure 2. Schematic of the measurement protocol for high precision Ne analysis using the Thermo Fisher ARGUS VI mass spectrometer. The analysis was performed in four lab books in Qtegra to minimise magnet current movement when $\mathrm{Ne}$ isotopes were measured. Interfering compounds and hydrogen were measured first for $10 \mathrm{~min}$. Ne isotope measurements were long enough to overcome the uncertainty introduced by the time gap.

\section{Cosmogenic Ne in Extra-Terrestrial Material: Multi-Collection Faraday Technique}

The multi-collection Faraday mode was developed for the determination of the ${ }^{21} \mathrm{Ne} /{ }^{20} \mathrm{Ne}$ of air [6] and precise analysis of the Ne isotope composition of natural gases [14]. The high sensitivity of the ARGUS system allowed this procedure to be used to determine cosmogenic Ne in small samples of extra-terrestrial material. Here, we report Ne isotopes in small samples (4.1 to $10.7 \mathrm{mg}$ ) of Bruderheim L-chondrite and the Imilac pallasite in order to establish the precision of $\mathrm{Ne}$ isotope determinations in meteorites.

Repeated analysis of air standard $(n=26)$ over the two-week analysis period yielded repeatability values of ${ }^{21} \mathrm{Ne} /{ }^{20} \mathrm{Ne}$ and ${ }^{22} \mathrm{Ne} /{ }^{20} \mathrm{Ne}$ of $0.17 \%$ and $0.09 \%$, respectively $(1 \sigma)$, calculated by fitting a best Gaussian curve to the probability density distribution [15]. All isotope data are consistent with the combination of mass fractionation of air and the presence of $\mathrm{NeH}^{+}$, as described earlier [6]. The repeatability of the intensity of ${ }^{20} \mathrm{Ne}$ ( $\sim 3450 \mathrm{fA}$ ) was $0.32 \%$. Isobaric interferences from ${ }^{40} \mathrm{Ar}^{2+}$ and ${ }^{44} \mathrm{CO}_{2}{ }^{2+}$ were trivial (less than $0.3 \%$ ) and all other interferences were significantly smaller than $0.1 \%$ [6].

The meteorite samples were heated to $1500{ }^{\circ} \mathrm{C}$ for $10 \mathrm{~min}$ in a double-walled furnace. The gas purification and $\mathrm{Ne}$ isotope analysis were conducted using methods described earlier [16]. Furnace hot blanks were measured and the Ne isotopic composition was found to be isotopically indistinguishable from air, albeit less than $0.3 \%$ of sample beam intensities. All samples were reheated, which confirmed that all $\mathrm{Ne}$ was extracted in the heating step. For these analyses, we did not routinely monitor $\mathrm{H}_{2} \mathrm{O}^{+}$and $\mathrm{HF}^{+}$as 
beam intensities were so low that it had a negligible $(<0.1 \%$ o effect on $m / z=20$. While ${ }^{20} \mathrm{NeH}^{+}$generation in the mass spectrometer ion source was significant for analyses of terrestrial $\mathrm{Ne}$ at partial pressures that are similar to those determined here, in this study, the contribution at $m / z=21$ was insignificant as ${ }^{21} \mathrm{Ne}^{+}$abundances in meteorites were approximately 30 times higher. We estimate that the ${ }^{20} \mathrm{NeH}^{+}$contribution for ${ }^{21} \mathrm{Ne}^{+}$was less than $0.1 \%$ in the meteorite analyses reported here.

The Ne isotope data for both meteorite samples are presented in Table 1 and Figure 3. The two Bruderheim samples yielded ${ }^{20} \mathrm{Ne} /{ }^{22} \mathrm{Ne}$ of $0.867 \pm 0.002$ and $0.852 \pm 0.002$ and ${ }^{21} \mathrm{Ne} /{ }^{22} \mathrm{Ne}$ values of $0.916 \pm 0.002$ and $0.925 \pm 0.002$. These plotted within the range determined by earlier studies ([15,16], (T. Graf, pers. comm.)), thus confirming our ability to replicate existing data. The difference in ${ }^{21} \mathrm{Ne} /{ }^{20} \mathrm{Ne}$ ratios of the two samples was small, but beyond the analytical uncertainty of each measurement. This may reflect variation in the contribution of the primordial Ne component within the samples. The Bruderheim sample had a well-established ${ }^{22} \mathrm{Ne}$ concentration of $12.14 \pm 0.11 \times 10^{-8} \mathrm{~cm}^{3} \mathrm{STP} / \mathrm{g}$ (personal communication with T. Graf). On this basis, using our measurements, we calculated an instrument sensitivity of $5.01 \pm 0.06 \times 10^{-12} \mathrm{~cm}^{3} \mathrm{STP} \mathrm{Ne} / \mathrm{fA}$, which was equivalent to $1.25 \pm 0.02 \times 10^{15} \mathrm{cps} / \mathrm{cm}^{3} \mathrm{STP}\left(\right.$ where $\left.1 \mathrm{cps}=1.6 \times 10^{-19} \mathrm{~A}\right)$.

The isotopic compositions of Ne for the two samples of Imilac pallasite were indistinguishable from each other. The ${ }^{20} \mathrm{Ne} /{ }^{22} \mathrm{Ne}$ values were $0.896 \pm 0.002$ and $0.893 \pm 0.002$ and the ${ }^{21} \mathrm{Ne} /{ }^{22} \mathrm{Ne}$ values were $1.028 \pm 0.003$ and $1.030 \pm 0.003$. These values were extremely close to those determined earlier for the Imilac pallasite [17] (Figure 3). The measured ${ }^{22} \mathrm{Ne}$ concentrations of $66.52 \pm 0.08$ and $69.42 \pm 0.07 \times 10^{-8} \mathrm{~cm}^{3} \mathrm{STP} / \mathrm{g}$ overlapped that of earlier determinations $\left(66.0 \pm 3.6 \times 10^{-8} \mathrm{~cm}^{3} \mathrm{STP}^{22} \mathrm{Ne} / \mathrm{g}\right)$ [17].

The precision of the Ne isotope ratios of both meteorites was $\pm 0.2-0.3 \%$. This was $\sim 50$ 100 and 5-10 times greater than the precision of ${ }^{20} \mathrm{Ne} /{ }^{22} \mathrm{Ne}$ and ${ }^{21} \mathrm{Ne} /{ }^{22} \mathrm{Ne}$ measurements of air, and was slightly higher than the repeatability of the air measurements $(0.17 \%$ and $0.09 \%$ for ${ }^{20} \mathrm{Ne} /{ }^{22} \mathrm{Ne}$ and ${ }^{21} \mathrm{Ne} /{ }^{22} \mathrm{Ne}$, respectively). We explain this by the large amount of matrix present in the gas phase after melting of the mineral. The intensity determinations had an uncertainty of $\pm 0.02 \%$ at signal sizes of $200 \mathrm{fA}$, and this was identical to the precision of ${ }^{22} \mathrm{Ne}$ beams of a similar size ( $\left.335 \mathrm{fA}\right)$ derived from air calibrations.

Table 1. Neon isotope data of newly measured meteorites.

\begin{tabular}{ccccc}
\hline Sample & Weight (mg) & ${ }^{\mathbf{2 0}} \mathbf{N e}{ }^{\mathbf{2 2}} \mathbf{N e}$ & ${ }^{{ }^{\mathbf{2 1}} \mathbf{N e} /{ }^{\mathbf{2 2}} \mathbf{N e}}$ & ${ }^{22} \mathbf{N e}$ \\
\hline Bruderheim-1 & 9.13 & This study & \\
Bruderheim-2 & 10.67 & $0.867(2)$ & $0.916(2)$ & N/A \\
\hline Imilac-1 & 10.10 & $0.852(2)$ & $0.925(2)$ & N/A \\
Imilac-2 & 4.10 & $1.028(3)$ & $0.893(2)$ & $69.52(8)$ \\
\hline \multicolumn{5}{c}{ Pers. comm. with T. Graf } \\
\hline Bruderheim-1 & 49.2 & 0.914 & 0.851 & 11.98 \\
Bruderheim-2 & 42.2 & 0.914 & 0.850 & 12.30 \\
Bruderheim-3 & 25.5 & 0.911 & 0.844 & 12.18 \\
Bruderheim-4 & 33.0 & 0.912 & 0.842 & 12.15 \\
Bruderheim-5 & 24.3 & 0.912 & 0.849 & 11.99 \\
Bruderheim-6 & 53.7 & 0.918 & 0.839 & 12.12 \\
Bruderheim-7 & 32.8 & 0.922 & 0.828 & 12.21 \\
Bruderheim-8 & 33.4 & 0.925 & 0.839 & \\
\hline
\end{tabular}

$1 \sigma$ are shown in parenthesis as last significant figures. Ne isotope concentrations are in $\mathrm{cm}^{3} \mathrm{STP} / \mathrm{g} \times 10^{8}$ where $p=0.101 \mathrm{MPa}$ and $\mathrm{T}=0{ }^{\circ} \mathrm{C}$ in accordance with [20]. N/A: not applicable as the Bruderheim, in this study, was used to determine mass spectrometer sensitivity using data from T. Graf (pers. comm.). Errors of data obtained from pers. comm. with T. Graf were not provided. 


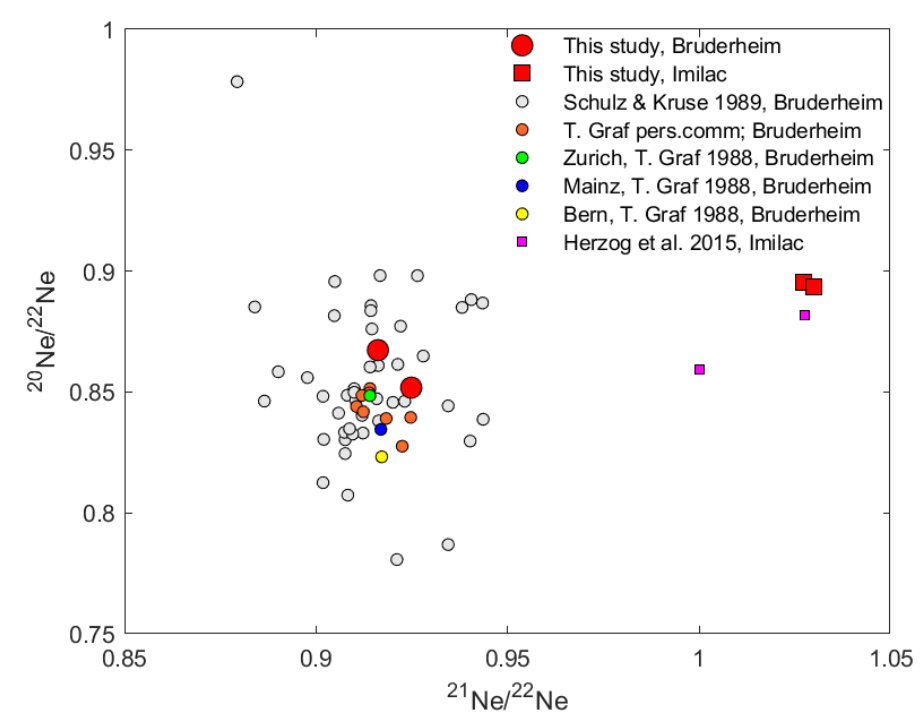

Figure 3. Neon isotopic composition of Bruderheim chondrite and Imilac pallasite analysed using ARGUS VI mass spectrometer. Bruderheim data overlapped with earlier determinations. Slight deviations in between the measurements may indicate variation in the contribution of primordial components. Imilac data overlapped with each other within the measurement error for both isotope ratios, and overlapped with earlier determinations. Measurement uncertainties are smaller than symbols. Other data are from Refs. [17-19] and personal communication with T. Graf.

Our data were obtained from a significantly smaller sample than earlier studies. The direct comparison of the quality of our data to earlier determinations is difficult because of the low number of analyses and the absence of precision in previous works. Our Bruderheim chondrite samples $(9.1$ and $10.7 \mathrm{mg}$ ) were between 2 and 6 times smaller than most recent measurements (T. Graf pers. comm.). Our Imilac pallasite samples (4.1 and $10.1 \mathrm{mg}$ ) were at least half the size of earlier determinations [17]. Incorporating the repeatability of air $\mathrm{Ne}$ isotope ratios into the overall isotope ratio uncertainty $(\sim 0.5 \%)$, we found a four-fold improvement on those measured in samples that were of at least 2-5 times greater mass [17].

\section{Cosmogenic Ne in Terrestrial Material: Analysis by Peak Jumping Using Electron Multiplier}

\subsection{Analysis Procedure and Repeated Measurement of Low Quantities of Atmospheric Ne}

The concentration of $\mathrm{Ne}$ in terrestrial rocks and minerals is significantly less than in meteorites, and typically requires the use of electron multiplier detectors [8-10]. We developed a peak jumping protocol using the CDD detector that was located in the L3 position. The peak measurement sequence followed that described above (Figure 2). Hydrogen $(m / z=2)$ and background $(m / z=2.2)$ were measured on the L2 Faraday detector with all other isotopes determined on the $\mathrm{CDD}$. $\mathrm{No} \mathrm{NeH}^{+}$was recorded during analysis, in line with findings using a Noblesse-HR at a similarly low Ne partial pressure [7].

Over the course of 4 months, the blanks were composed of fractionated air $(n=36)$. Isobaric interference from $\mathrm{HF}^{+}$always stayed below $1 \%$. Contributions at $m / z=20$ from $\mathrm{H}_{2}{ }^{18} \mathrm{O}^{+}(\sim 1 \%)$ and ${ }^{40} \mathrm{Ar}^{++}(\sim 5 \%)$ were small. In contrast, ${ }^{44} \mathrm{CO}_{2}{ }^{++}$dominated $\mathrm{m} / z=22$. The air-like blank in the mass spectrometers rendered blank correction for cosmogenic $\mathrm{Ne}$ redundant.

Calibration data $(n=103)$ were collected over the course of 4 months from aliquots of air at the level of $9.36 \pm 0.21 \times 10^{-14} \mathrm{~cm}^{3} \mathrm{STP}^{21} \mathrm{Ne}$, which was equivalent to ${ }^{21} \mathrm{Ne}$ from $7.7 \mathrm{mg}$ CREU quartz. The repeatability of the ${ }^{21} \mathrm{Ne} /{ }^{20} \mathrm{Ne}$ and ${ }^{22} \mathrm{Ne} /{ }^{20} \mathrm{Ne}$ of air was $1.10 \%$ and $0.62 \%$, respectively (Figure 4$)(1 \sigma, 2$ outliers), which was calculated by fitting a best-fit Gaussian curve to the probability density distribution. The isobaric interferences from $\mathrm{H}_{2} \mathrm{O}^{+}, \mathrm{HF}^{+}$and ${ }^{40} \mathrm{Ar}^{2+}$ at $m / z=20,{ }^{63} \mathrm{Cu}^{3+}$ at $m / z=21$ and ${ }^{66} \mathrm{Zn}^{3+}$ at $m / z=22$ were trivial. 
Interference from organic compounds was assumed to be negligible [6]. The contribution of $\mathrm{CO}_{2}{ }^{2+}$ at $m / z=22$ averaged around $7 \%$ over the 4-month period. Singly/doubly charged component determinations followed earlier practice [6]. The isotope ratios plotted slightly to the right of the mass fractionation line in Figure 4, indicating either a constant excess at $m / z=21$ (termed excess Ne) (horizontal movement in Figure 4), which does not appear in the blank measurements, or a different $\mathrm{CO}_{2}$ contribution to that of the dynamic blanks (vertical movement in Figure 4). However, doubly charged $\mathrm{CO}_{2}$ would only increase with increasing partial pressure in the mass spectrometer [13,21], which would move our data to the lower ${ }^{22} \mathrm{Ne} /{ }^{20} \mathrm{Ne}$ regimes and would not explain our data. Therefore, we suggest the presence of excess $\mathrm{Ne}$.

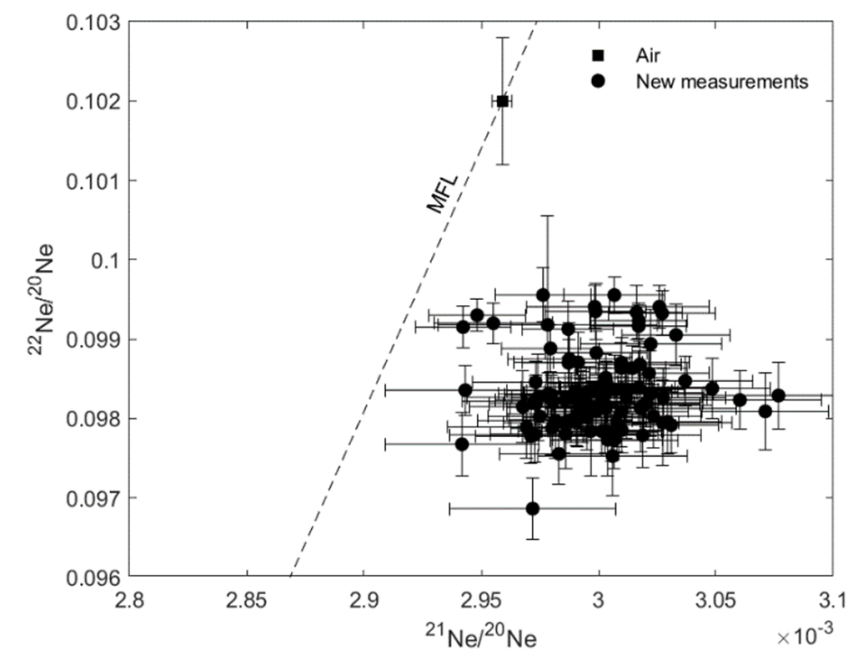

Figure 4. Ne isotope ratio from the repeated analysis of air using the ARGUS VI mass spectrometer. The delivery amount was $9.36 \pm 0.21 \times 10^{-14} \mathrm{~cm}^{3} \mathrm{STP}{ }^{21} \mathrm{Ne} /$ aliquot, equivalent of ${ }^{21} \mathrm{Ne}$ from $7.7 \mathrm{mg}$ CREU. The repeatability of ${ }^{21} \mathrm{Ne} /{ }^{20} \mathrm{Ne}$ was $1.1 \%(1 \sigma)(\mathrm{n}=103)$, which was a significant achievement at this partial pressure of Ne. The repeatability of ${ }^{22} \mathrm{Ne} /{ }^{20} \mathrm{Ne}$ was $0.62 \%$ and was likely governed by the $7 \% \mathrm{CO}_{2}$ correction at mass 22 . All data were located slightly right of the mass fractionation line (MFL), indicating a constant excess at $m / z=21$, which was unchanged over an order of magnitude of Ne partial pressure and was corrected out (see text). Air is from Refs. [6,22]. Plotted uncertainties are $1 \sigma$.

Excess $\mathrm{Ne}$, or, more precisely, excess mass 21 is the result of the liberation of material inside the mass spectrometer by the calibration (or sample) beam. This was not detected on the Faraday detectors at higher beam intensities, and the fact that the CDD is known to produce $\mathrm{CO}_{2}$ implies that the electron multiplier is the source [6]. Test measurements revealed that the excess remains unchanged over an order of magnitude of Ne partial pressure in the mass spectrometer. This suggests that if the sample stays within this partial pressure range, the excess can simply be corrected out. In the worst-case scenario when the sample is outside of this range, which was not the case for this study, our accuracy could vary by the extent of the excess-thus, by at least $\pm 1 \%$ - and would leave the precision unchanged. Intensities of ${ }^{20} \mathrm{Ne}$ (i.e., sensitivity) were found to be variable in the short-term (days/weeks), but long-term (4 months) observation suggests that the variability is natural. ${ }^{20}$ Ne reproduced by $1.13 \%$ (9 outliers out of $103,1 \sigma$ ) over this period (Figure 5 ). We suggest this this should be used as the best representation of the repeatability of a sample. The best two-week period exhibited repeatability that was as low as $0.16 \%(n=10)$, obtained from calibration data acquisition with blank measurements before and after each, with no interruption of the pumping and baking laser pan volume and subsequent sample analysis. 


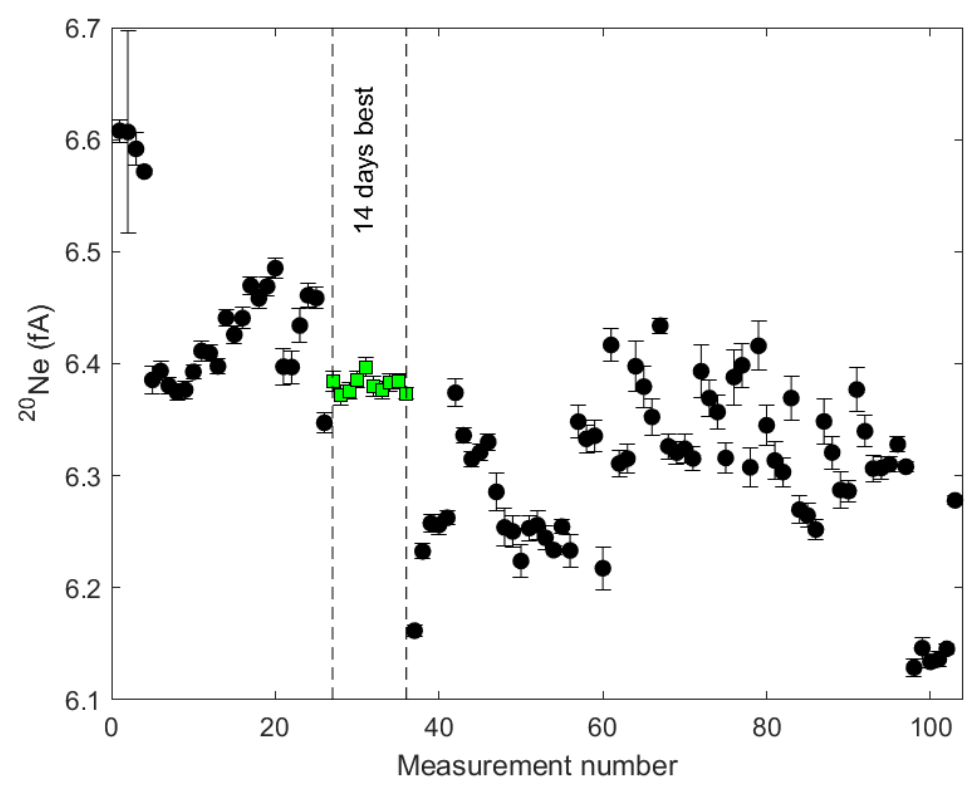

Figure 5. ${ }^{20} \mathrm{Ne}$ intensities from the repeated analysis of $3.2 \times 10^{-11} \mathrm{~cm}^{3} \mathrm{STP}{ }^{20} \mathrm{Ne}$ of air using the ARGUS VI mass spectrometer. Repeatability over the 4 months analytical period was $1.13 \%$ (ignoring 9 outliers out of 103). The best 14-day period (green squares) $(n=10)$ exhibited a repeatability of $0.16 \%$ when no interruption of sample analysis occurred. The ${ }^{21} \mathrm{Ne}$ concentration of air/calibration aliquot was equivalent to that of $7.7 \mathrm{mg}$ CREU-1 quartz. Long-term variability seems to be the natural variability of sensitivity, which we think is the best representation of the repeatability of unknowns.

\subsection{CREU-1 Quartz}

Samples of $\sim 19.9 \mathrm{mg}$ of $250-500 \mu \mathrm{m}$ CREU-1 quartz [5] were weighed in $>99 \%$ pure, $20 \times 2 \mathrm{~mm}$ Pt foil tubes. The tubes were crimped at both ends then placed into $1 \mathrm{~cm}^{2}$ recesses in a fully degassed $\mathrm{Cu}$ pan and pumped to $<10^{-8}$ mbar prior to degassing at $80^{\circ} \mathrm{C}$ for $12 \mathrm{~h}$. A sapphire cover glass was used to avoid volatilized metal from adsorbing onto the sapphire viewport. Neon was extracted from the samples by heating to $\sim 1350{ }^{\circ} \mathrm{C}$ for 10 min using a 75 W Fusions 970 (Photon Machines) diode laser $(970 \mathrm{~nm}$ ) [23]. Remote operation of the laser was developed during the COVID-related lockdown in late 2020. This, combined with automated gas purification and separation [6], allowed full remote analysis of 10 samples (one laser pan) without the need for laboratory attendance. This significantly increased sample throughput to around 20 samples (2 laser pans) per week and had the advantage that samples could be analysed more closely in time, thereby eliminating slight fluctuations in sensitivity (Figure 5). The cold blanks of the laser pan and the heat of the empty Pt tube (hot blank) were indistinguishable from the system blanks. Isobaric interferences were corrected as above and were similar to air calibrations.

All CREU quartz yielded Ne isotope data plotted on or close to the established spallation line (Figure 6A,B). The ${ }^{21} \mathrm{Ne} /{ }^{20} \mathrm{Ne}$ and ${ }^{22} \mathrm{Ne} /{ }^{20} \mathrm{Ne}$ ratios varied from $0.00836 \pm 0.00007$ to $0.01379 \pm 0.00022$ and from $0.1092 \pm 0.00036$ to $0.1145 \pm 0.00063$, respectively. The samples contained higher proportions of air than most samples reported in the international calibration exercise $[5,24,25]$. This may reflect the low temperature and short duration of the pre-extraction bake out. 


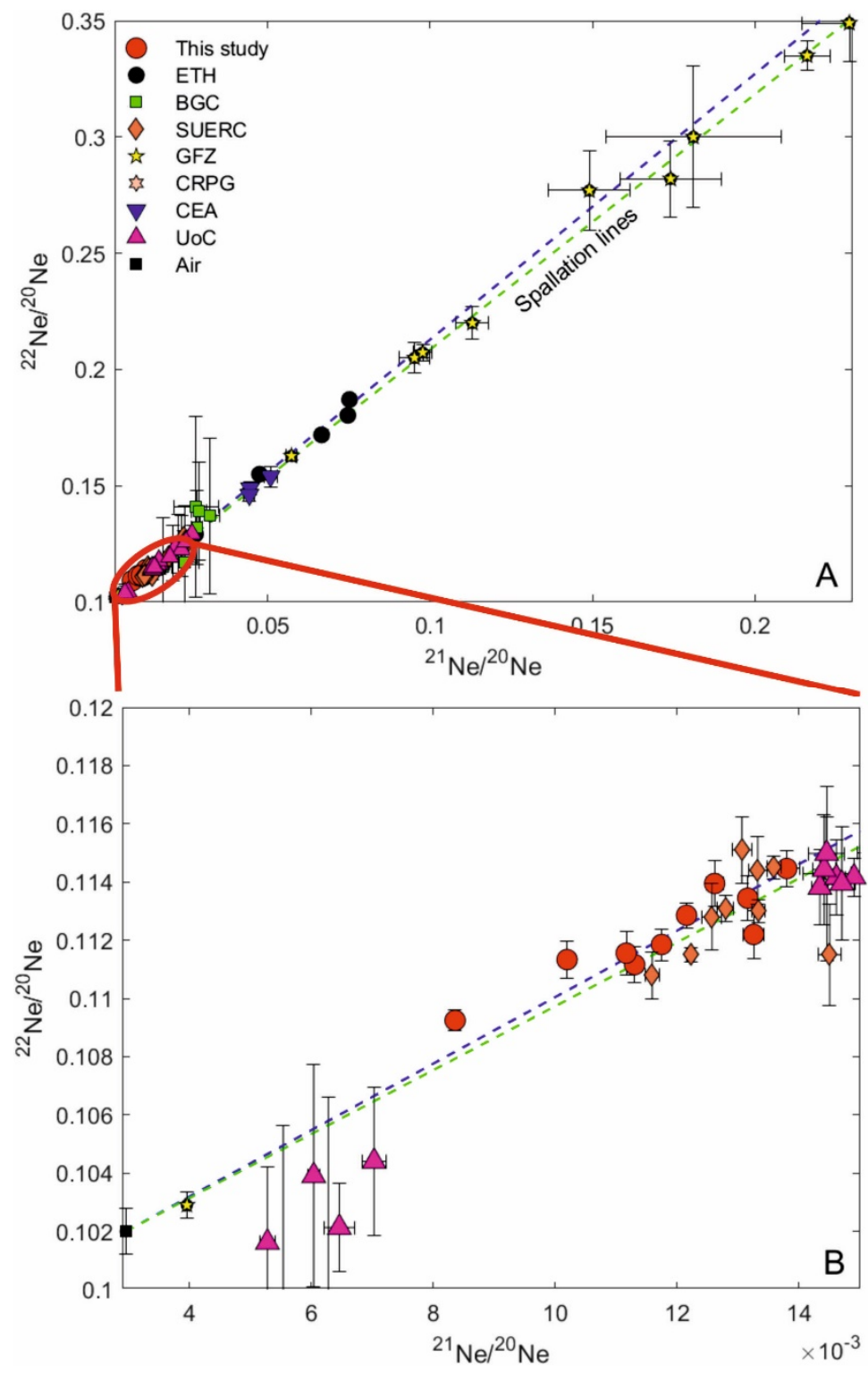

Figure 6. Neon isotope composition of $19.9 \mathrm{mg}$ CREU-1 quartz using the ARGUS VI mass spectrometer. Data were plotted on or close to established cosmogenic spallation lines (A) ([26] blue and [27] green), with small deviations that were similar to earlier determinations (B). Data were produced over 4 months. Air value (black square) from [6,22]. Other data are from $[5,24,25]$. ETH (Switzerland), BGC (USA), SUERC (UK), GFZ (Germany), CRPG (France), CEA: China Earthquake Administration (PR China), UoC: University of Cologne (Germany).

The quality of the ${ }^{21} \mathrm{Ne} /{ }^{20} \mathrm{Ne}$ measurements of CREU quartz, which is the basis for calculating the cosmogenic ${ }^{21} \mathrm{Ne}$ content, is now being assessed against the values obtained in other laboratories. The measurement error is governed by the signal of ${ }^{21} \mathrm{Ne}$, which is governed by two factors: (1) the degree of cosmogenic compound in the mineral versus atmospheric compound (e.g., how far the sample was located from air on the spallation line) and (2) the mass of the mineral analysed. Consequently, theory would suggest that a higher ${ }^{21} \mathrm{Ne}$ signal (via one of the two factors given above) would lead to a smaller relative error of ${ }^{21} \mathrm{Ne} /{ }^{20} \mathrm{Ne}$. Unfortunately, when data were plotted on a 3D plot of the relative error of ${ }^{21} \mathrm{Ne} /{ }^{20} \mathrm{Ne}$ and ${ }^{21} \mathrm{Ne} /{ }^{20} \mathrm{Ne}$, and the mass of CREU, we were unable to conclude this (Figure 7). We noticed no trend in the error with either increasing mass or ${ }^{21} \mathrm{Ne} /{ }^{20} \mathrm{Ne}$ and, at this stage, we were unable to compare our performance to that of other laboratories. 
We suggest that there is at least one more factor that we have to take into account in order to assess our performance against that of other laboratories.

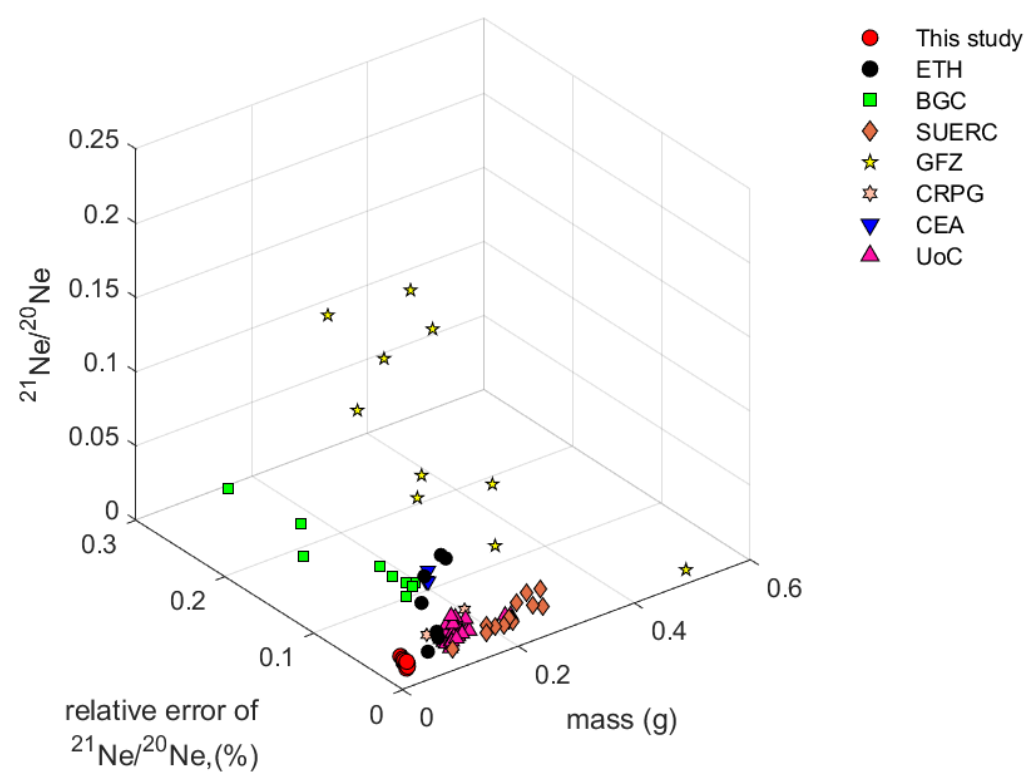

Figure 7. The error of ${ }^{21} \mathrm{Ne} /{ }^{20} \mathrm{Ne}$ from CREU-1 quartz with respect to the absolute isotope ratio value and amount of material analysed (mass) from a number of different laboratories. In theory, the relative error $(1 \sigma)$ should decrease with either increasing mass or increasing ${ }^{21} \mathrm{Ne} /{ }^{20} \mathrm{Ne}$ or the combination of both. No trend of this nature was observed, suggesting that other factors govern the relative error in the Ne isotope ratio of CREU. ETH (Switzerland), BGC (USA), SUERC (Scotland), GFZ (Germany), CRPG (France): CEA: China Earthquake Administration (PR China), UoC: University of Cologne (Germany). Data are from Refs. [5,24,25].

Using the established procedure [13], the cosmogenic ${ }^{21} \mathrm{Ne}$ content of the CREU1 quartz samples ranged from 2.91 to $3.73 \times 10^{8}$ atoms $/ \mathrm{g}$, with the majority having a much narrower range from 3.08 to $3.50 \times 10^{8}$ atoms $/ g$ (Table 2). The mean cosmogenic ${ }^{21} \mathrm{Ne}$ concentration, calculated by fitting the best Gaussian curve (see above), was $3.25 \pm 0.24 \times 10^{8}$ atoms $/ g(2 \sigma, 2$ outliers). While this was $6.6 \%$ lower than the accepted value of $3.48 \pm 10 \times 10^{8}$ [5], it overlapped with $2 \sigma$. Further, it overlapped with the data from SUERC and GFZ, it was indistinguishable with the data reported by CRPG [5], and the mean value differed from the arithmetic mean to the same extent as the average BGC data (Figure 8). Regarding the outliers, we explain the highest value $\left(3.73 \times 10^{8}\right.$ atoms $\left./ g\right)$ by a memory effect in the system caused by inadequate pumping due to a failure of the pump for an unknown period of time overnight. The error on ${ }^{21} \mathrm{Ne}^{*}$ was estimated using the 4-month calibration data. As discussed above, we think this is the best representation of instrument performance and avoids underestimation of our error.

These data were obtained from $19.9 \pm 0.5 \mathrm{mg}$ CREU- 1 quartz, and constituted, by a significant margin, the smallest amount yet reported (Table 2). Previously published measurements of $<250 \mathrm{mg}$ aliquots of CREU-1 quartz $[5,24,25]$ binned in $10 \mathrm{mg}$ groups for each laboratory are plotted in Figure 9. It can be seen that twenty one data groups were produced if we ignore data from the fine fraction from ref. [5] (Table S1). We applied the same statistics as above and ignored groups that would be made up by one data point only. The exception from this is CRPG data, which showed a significant underdispersion.

The cosmogenic ${ }^{21} \mathrm{Ne}$ generated by measurement in early generation static vacuum magnetic-sector mass spectrometers (VG5400 (CRPG, GFZ, CEA) and MAP 215-50 (SUERC)) showed repeatability of between $4 \%(80 \mathrm{mg})$ and $2.1 \%$ (>200 mg). Data obtained from state-of-the-art analytical systems, e.g., Thermo Fisher ARGUS VI (this study), MAP 215-50 with modern electronics (BGC) and Helix MC Plus (UoC), exhibited a significant 
improvement. The data (39 measurements incorporated into 9 groups) appeared to plot along an exponential curve. They implied that modern instrumentation generates a 5-fold improvement in repeatability compared to data produced by VG5400 and MAP 215-50 instruments. This improvement was the result of the combination of several factors. The development of new source electronics led to more stable ion sources. New control software (e.g., Qtegra in the case of the Thermo Fisher mass spectrometers) with built-in regression functions replaced in-house built data manipulation software for old generation mass spectrometers, which resulted in two key outcomes: (1) the generalization of a regression method with improved mathematics and error propagation, and (2) the opportunity for computer-controlled, automatized gas preparation. The latter means more precise reproduction of gas preparation (i.e., opening and closing valves with highly precise time intervals in between steps) and more precise temperature control of cold fingers. We were unable to resolve the question as to which of these factors played the most significant role in improving the repeatability of Ne isotope determinations. The custom-built mass spectrometer at ETH [28] showed the best performance, although one data point was able to fit the exponential model of repeatability and mass of the previous group, but this is not commercially available.

Table 2. Ne data of CREU quartz analysed using SUERC's ARGUS VI mass spectrometer.

\begin{tabular}{ccccc}
\hline Sample & Weight $(\mathbf{m g})$ & ${ }^{\mathbf{2 1}} \mathbf{N e}{ }^{\mathbf{2 0}} \mathbf{N e}$ & ${ }^{22} \mathbf{N e}{ }^{\mathbf{2 0}} \mathbf{N e}$ & ${ }^{\mathbf{2 1}} \mathbf{N e}^{*}$ \\
\hline CREU-A & 19.67 & $0.0084(1)$ & $0.1092(4)$ & $332(6)$ \\
CREU-B & 19.45 & $0.0102(1)$ & $0.1113(6)$ & $329(6)$ \\
CREU-C & 19.36 & $0.0132(1)$ & $0.1134(8)$ & $373(7)$ \\
CREU-D & 20.08 & $0.0122(1)$ & $0.1129(4)$ & $350(7)$ \\
CREU-E & 20.88 & $0.0117(1)$ & $0.1119(5)$ & $323(6)$ \\
CREU-F & 20.29 & $0.0138(2)$ & $0.1145(6)$ & $327(6)$ \\
CREU-G & 19.64 & $0.0133(2)$ & $0.1122(8)$ & $308(6)$ \\
CREU-H & 19.98 & $0.0126(1)$ & $0.1139(8)$ & $318(6)$ \\
CREU-I & 19.18 & $0.0113(1)$ & $0.1112(6)$ & $324(6)$ \\
CREU-J & 19.48 & $0.0112(1)$ & $0.1116(7)$ & $291(5)$
\end{tabular}

$1 \sigma$ errors are shown in parenthesis as last significant figures. ${ }^{21} \mathrm{Ne}^{*}$ is the cosmogenic (non-atmospheric) ${ }^{21} \mathrm{Ne}$. Concentration is given in $\mathrm{cm}^{3} \mathrm{STP} / \mathrm{g} \times 10^{6}$, where $p=0.101 \mathrm{MPa}$ and $\mathrm{T}=0{ }^{\circ} \mathrm{C}$ in accordance with [20].

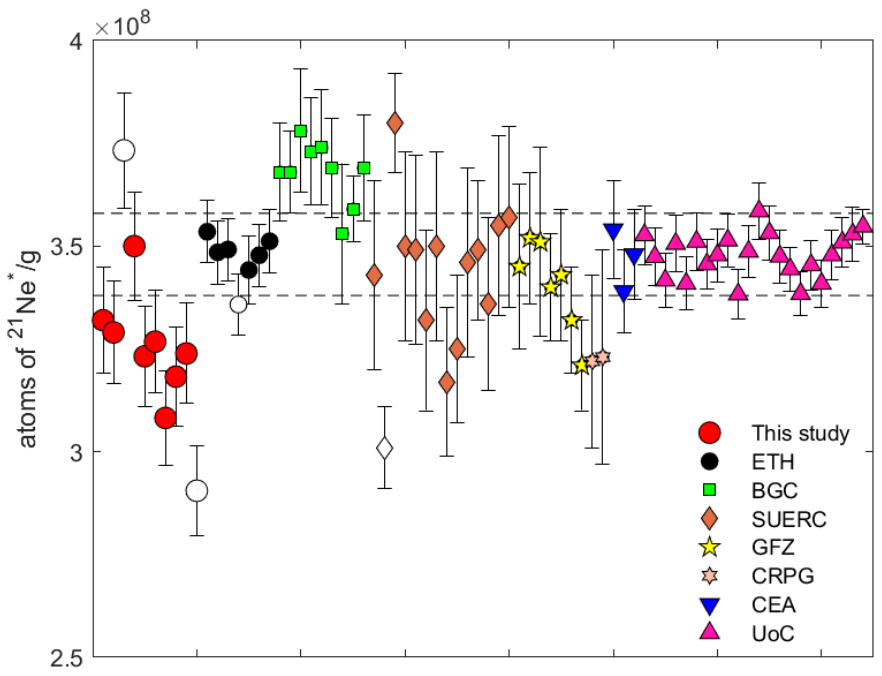

Figure 8. Cosmogenic ${ }^{21} \mathrm{Ne}$ in CREU-1 quartz measured using the ARGUS VI mass spectrometer. Mean value $\left(3.25 \pm 0.12 \times 10^{821} \mathrm{Ne}^{*}\right.$ atoms $/ \mathrm{g}, \mathrm{n}=8,2$ outliers) was lower by $6.6 \%$ than the accepted standard value (dashed line, $348 \pm 10 \times 10^{821} \mathrm{Ne}^{*}$ atoms $/ \mathrm{g}$ ) but it overlapped it within $2 \sigma$. Data are after Refs. [5,24,25]. ETH (Switzerland), BGC (USA), SUERC (Scotland), GFZ (Germany), CRPG (France): CEA: China Earthquake Administration (PR China), UoC: University of Cologne (Germany). 2 sigma uncertainties are plotted. Empty: outlier. 


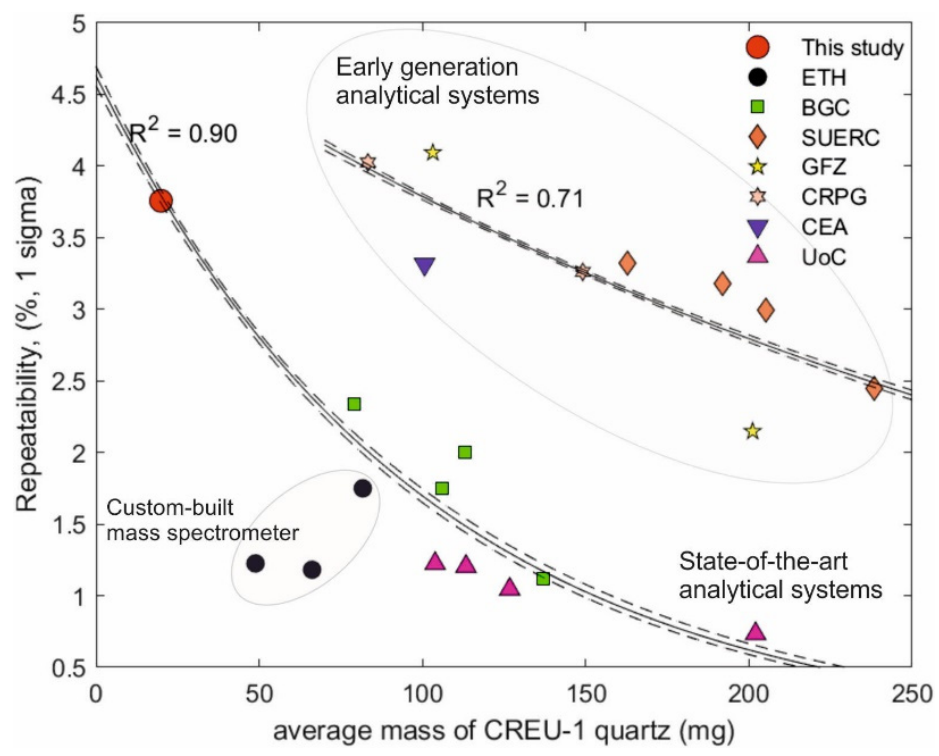

Figure 9. Repeatability of CREU-1 quartz measurements with respect to analysed amounts from a number of different laboratories worldwide. Data from Refs. [5,24,25] were split into groups by masses within $10 \mathrm{mg}$ of material. The only exception was CPRG, which were significantly under dispersed. Data show that beside analysed mass (e.g., signal size of ${ }^{21} \mathrm{Ne}$ ) the analytical system is a key factor in data quality. Data from early generation analytical systems (SUERC, CRPG, GFZ, CEA) grouped together and showed the lowest performance at a given mass. Data emerged from state-of-the-art analytical systems including modern electronics, and a high degree of automation (see text) (ARGUS VI in SUERC of this study, BGC and UoC) characterises another group, showing an excellent correlation ( $n=9,39$ individual measurements) between the analysed amount of CREU-1 quartz and of ${ }^{21} \mathrm{Ne}^{*}$. Only the custom-built mass spectrometer (ETH) showed a better performance. We conclude that SUERC's ARGUS VI mass spectrometer analysed $19.9 \mathrm{mg}$ of CREU-1 quartz performs as expected. Apart from three laboratories globally (ETH, BGC and UoC), all laboratories would require the analysis of 5 times or more material to reach the repeatability of this study. ETH (Switzerland), BGC (USA), SUERC (Scotland), GFZ (Germany), CRPG (France): CEA: China Earthquake Administration (PR China), UoC: University of Cologne (Germany). 1 sigma errors on masses are smaller than symbols. Curve errors are 1 sigma.

\section{Conclusions and Future Research Directions}

The Thermo Fisher ARGUS VI mass spectrometer, tuned for high precision Ne analysis, is now well characterised for the determination of cosmogenic $\mathrm{Ne}$ in terrestrial and extraterrestrial rocks. We characterised the performance in both multi-collection Faraday and peak jumping mode using an electron multiplier. In multi-collection Faraday mode, we replicated the Ne isotope composition of Bruderheim L chondrite and Imilac pallasite. For extra-terrestrial material, we found a 4-fold improvement in the overall uncertainty of the Ne isotope ratio $(0.5 \%)$ compared to that obtained using 2-6 times more material in earlier works. We suggest that a 5-10-fold reduction in the repeatability may be obtained, albeit more measurement would be needed to confirm this. Peak jumping on the CDD detector allowed the analysis of cosmogenic Ne in 19.9 mg CREU-1 quartz, which was 3-5 times less than ever reported in previous studies and the acquisition of calibration data the level of $9.36 \pm 0.21 \times 10^{-14} \mathrm{~cm}^{3} \mathrm{STP}{ }^{21} \mathrm{Ne}$ (equivalent of $7.7 \mathrm{mg}$ CREU). We reproduced ${ }^{21} \mathrm{Ne} /{ }^{20} \mathrm{Ne}$ with $1.1 \%(1 \sigma)(\mathrm{n}=103)$, which was a significant achievement at this Ne partial pressure. Our CREU measurement yielded to ${ }^{21} \mathrm{Ne}^{*}$ content of $325 \pm 12 \times 10^{621} \mathrm{Ne}^{*}$ atoms/g $(\mathrm{n}=8)$, which overlaps with the internationally accepted value within $2 \sigma$. The reproducibility of ${ }^{21} \mathrm{Ne}^{*}(3.7 \%, 1$ sigma) was exactly what the relationship of repeatability and sample mass would suggest, established by data obtained from similar, state-of-the-art analytical systems. This was a $\sim 4$ times improvement in comparison to early generation 
analytical systems. The remote operation of laser heating and the automation of gas purification, separation and analysis procedures increased sample throughput and exact repetition of the procedure and, by allowing more calibration and blank measurements, would likely produce better quality data. The ability to determine Ne isotopes in small amounts of material will be invaluable in studies where sample material is extremely limited, e.g., planetary and comet return missions, and when the appropriate mineral phase is in low abundances in terrestrial or extra-terrestrial rocks.

The incorporation of high gain Faraday amplifiers $\left(10^{13} \mathrm{Ohm}\right.$ and beyond) may allow further improvements in data quality and reductions in sample size for the analysis of cosmogenic Ne-rich material. However, ${ }^{21} \mathrm{Ne}$ analysis from terrestrial samples will likely remain beyond the reach of Faraday detectors with existing amplifier technology and mass spectrometer sensitivity. For small sample analysis, effort will focus on reducing the background level of $\mathrm{CO}_{2}$, which will prove difficult to resolve without the application of high-resolution instrumentation, e.g., that of [29], and the exploration of the advantages of multi-collection using combined CDD-Faraday.

Supplementary Materials: The following are available online at https:/ / www.mdpi.com/article/10.3 390 /geosciences11080353/s1, Table S1. Air calibration measurements from aliquots of $9.4 \times 10^{-14} \mathrm{~cm}^{3}$ STP $21 \mathrm{Ne}$.

Author Contributions: Conceptualization: F.M.S., D.G. and L.D.N. Analytical development: D.G. and L.D.N. Data acquisition: D.G., L.D.N. and D.C. Data interpretation: F.M.S., D.G., L.D.N. and D.C. First draft: D.G. and F.M.S. Writing and editing: all authors. All authors have read and agreed to the published version of the manuscript.

Funding: This research received no external funding.

Data Availability Statement: All data are available in Tables and Supplementary Materials.

Acknowledgments: The research has been funded by SUERC. Rainer Wieler is thanked for providing Bruderheim samples and Thomas Graf is thanked for providing unpublished Ne isotope data of Bruderheim. John Faithfull provided a sample of Imilac from the Hunterian Museum collection. Ángel Rodés is thanked for help in statistics.

Conflicts of Interest: The authors declare no conflict of interest.

\section{References}

1. Dunai, T.J.; González López, G.A.; Juez-Larré, J. Oligocene-Miocene age of aridity in the Atacama Desert revealed by exposure dating of erosion-sensitive landforms. Geology 2005, 33, 321-324. [CrossRef]

2. Füri, E.; Zimmermann, L.; Deloule, E.; Trappitsch, R. Cosmic ray effects on the isotope composition of hydrogen and noble gases in lunar samples: Insights from Apollo 12018. Earth Planet. Sci. Lett. 2020, 550, 116550. [CrossRef]

3. Sinclair, H.D.; Stuart, F.M.; Mudd, S.M.; McCann, L.; Tao, Z. Detrital cosmogenic 21Ne records decoupling of source-to-sink signals by sediment storage and recycling in Miocene to present rivers of the Great Plains, Nebraska, USA. Geology 2018, 47, 3-6. [CrossRef]

4. Wieler, R. Cosmic-Ray-Produced Noble Gases in Meteorites. Rev. Mineral. Geochem. 2002, 47, 125-170. [CrossRef]

5. Vermeesch, P.; Balco, G.; Blard, P.-H.; Dunai, T.J.; Kober, F.; Niedermann, S.; Shuster, D.L.; Strasky, S.; Stuart, F.M.; Wieler, R.; et al. Interlaboratory comparison of cosmogenic 21Ne in quartz. Quat. Geochronol. 2015, 26, 20-28. [CrossRef]

6. Györe, D.; Tait, A.; Hamilton, D.; Stuart, F.M. The formation of NeH+ in static vacuum mass spectrometers and re-determination of $21 \mathrm{Ne} / 20 \mathrm{Ne}$ of air. Geochim. Cosmochim. Acta 2019, 263, 1-12. [CrossRef]

7. Saxton, J.M. The $21 \mathrm{Ne} / 20 \mathrm{Ne}$ ratio of atmospheric neon. J. Anal. At. Spectrom. 2020, 35, 943-952. [CrossRef]

8. Farley, K.A.; Treffkorn, J.; Hamilton, D. Isobar-free neon isotope measurements of flux-fused potential reference minerals on a Helix-MC-Plus mass spectrometer. Chem. Geol. 2020, 537, 119487. [CrossRef]

9. Marrocchi, Y.; Burnard, P.G.; Hamilton, D.; Colin, A.; Pujol, M.; Zimmermann, L.; Marty, B. Neon isotopic measurements using high-resolution, multicollector noble gas mass spectrometer: HELIX-MC. Geochem. Geophys. Geosyst. 2009, 10. [CrossRef]

10. Honda, M.; Zhang, X.; Phillips, D.; Hamilton, D.; Deerberg, M.; Schwieters, J.B. Redetermination of the 21Ne relative abundance of the atmosphere, using a high resolution, multi-collector noble gas mass spectrometer (HELIX-MC Plus). Int. J. Mass Spectrom. 2015, 387, 1-7. [CrossRef]

11. Ruzié-Hamilton, L.; Clay, P.L.; Burgess, R.; Joachim, B.; Ballentine, C.J.; Turner, G. Determination of halogen abundances in terrestrial and extraterrestrial samples by the analysis of noble gases produced by neutron irradiation. Chem. Geol. 2016, 437, 77-87. [CrossRef] 
12. Bai, X.; Qiu, H.; Liu, W.; Mei, L. Automatic 40Ar/39Ar dating techniques using multicollector ARGUS VI noble gas mass spectrometer with self-made peripheral apparatus. J. Earth Sci. 2018, 29, 408-415. [CrossRef]

13. Niedermann, S.; Graf, T.; Marti, K. Mass spectrometric identification of cosmic-ray-produced neon in terrestrial rocks with multiple neon components. Geochim. Cosmochim. Acta 1993, 118, 65-73. [CrossRef]

14. Gilfillan, S.M.V.; Györe, D.; Flude, S.; Johnson, G.; Bond, C.E.; Hicks, N.; Lister, R.; Jones, D.G.; Kremer, Y.; Haszeldine, R.S.; et al. Noble gases confirm plume-related mantle degassing beneath Southern Africa. Nat. Commun. 2019, 10, 1-7. [CrossRef]

15. Kirkup, L. Data Analysis for Physical Scientists, Featuring Excel, 2nd ed.; Cambridge University Press: Cambridge, UK, 2012.

16. Ritter, B.; Stuart, F.M.; Binnie, S.A.; Gerdes, A.; Wennrich, V.; Dunai, T.J. Neogene fluvial landscape evolution in the hyperarid core of the Atacama Desert. Sci. Rep. 2018, 8, 13952. [CrossRef] [PubMed]

17. Herzog, G.F.; Cook, D.L.; Cosarinsky, M.; Huber, L.; Leya, I.; Park, J. Cosmic-ray exposure ages of pallasites. Meteorit. Planet. Sci. 2015, 50, 86-111. [CrossRef]

18. Graf, T. Produktion Kosmogener Nuklide in Meteoriten. Ph.D. Thesis, ETH Zürich, Zürich, Switzerland, 1988; 136p. (In German).

19. Schultz, L.; Kruse, H. Helium, neon and argon in meteorites-A data compilation. Meteoritics 1989, 24, 155-172. [CrossRef]

20. Ozima, M.; Podosek, F.A. Noble Gas Geochemistry, 2nd ed.; Cambridge University Press: Cambridge, UK, $2002 ;$ p. 367.

21. Balco, G.; Shuster, D.L. Production rate of cosmogenic $21 \mathrm{Ne}$ in quartz estimated from $10 \mathrm{Be}, 26 \mathrm{Al}$, and $21 \mathrm{Ne}$ concentrations in slowly eroding Antarctic bedrock surfaces. Earth Planet. Sci. Lett. 2009, 281, 48-58. [CrossRef]

22. Eberhardt, P.; Eugster, O.; Marti, K. A redetermination of the isotopic composition of atmospheric neon. Z. Nat. A 1965, 20, 623-624. [CrossRef]

23. Carracedo, A.; Rodés, Á.; Smellie, J.L.; Stuart, F.M. Episodic erosion in West Antarctica inferred from cosmogenic 3He and 10Be in olivine from Mount Hampton. Geomorphology 2019, 327, 438-445. [CrossRef]

24. Ritter, B.; Vogt, A.; Dunai, T.J. Noble gas extraction procedure and performance of the Cologne Helix MC Plus multi-collector noble gas mass spectrometer for cosmogenic neon isotope analysis. Geochronology 2021, 3, 421-431. [CrossRef]

25. Ma, Y.; Wu, Y.; Li, D.; Zheng, D. Analytical procedure of neon measurements on GV 5400 noble gas mass spectrometer and its evaluation by quartz standard CREU-1. Int. J. Mass Spectrom. 2015, 380, 26-33. [CrossRef]

26. Niedermann, S.; Graf, T.; Kim, J.S.; Kohl, C.P.; Marti, K.; Nishiizumi, K. Cosmic-ray-produced 21Ne in terrestrial quartz: The neon inventory of Sierra Nevada quartz separates. Earth Planet. Sci. Lett. 1994, 125, 341-355. [CrossRef]

27. Schäfer, J.M.; Ivy-Ochs, S.; Wieler, R.; Leya, I.; Baur, H.; Denton, G.H.; Schlüchter, C. Cosmogenic noble gas studies in the oldest landscape on earth: Surface exposure ages of the Dry Valleys, Antarctica. Earth Planet. Sci. Lett. 1999, 167, 215-226. [CrossRef]

28. Baur, H. Numerische Simulation und Praktische Erprobung einer Rotationssymmetrischen Ionenquelle für Gasmassenspektrometer. Ph.D. Thesis, ETH Zürich, Zürich, Switzerland, 1980. (In German).

29. Eiler, J.M.; Clog, M.; Magyar, P.; Piasecki, A.; Sessions, A.; Stolper, D.; Deerberg, M.; Schlueter, H.-J.; Schwieters, J. A highresolution gas-source isotope ratio mass spectrometer. Int. J. Mass Spectrom. 2013, 335, 45-56. [CrossRef] 